\title{
Poor Knowledge of Colorectal Cancer in Brunei Darussalam
}

\author{
Vui Heng Chong'*, Ai Giok Lim¹, Hana Naqiyah Baharudin ${ }^{1}$, Jackson Tan², \\ Chee Fui Chong ${ }^{3}$,
}

\begin{abstract}
Background: Colorectal cancer (CRC) is the most common gastrointestinal cancer and the incidence is increasing in many developing countries. While it can be detected early and even prevented through screening and removal of premalignant lesions, there are barriers to screening which include low level of knowledge and awareness of CRC. This study assessed the level of knowledge of CRC in Brunei Darussalam. Materials and Methods: A total of 431 (262 male and 161 female) subjects participated in this questionnaire study. Subjects were scored on their knowledge of signs/symptoms (maximum 10 correct answers) and known risk factors for CRC (maximum 10 correct answers) and were categorised into poor (0-2), moderate (3-4) and satisfactory (5-10). Comparisons were made between the various patient factors. Results: Overall, $54.1 \%$ could not name any CRC signs/symptoms or associated risk factors. Most were not aware of any screening modalities. The overall scores for CRC signs/symptoms and risk factors were $1.3 \pm 1.39$ (range $0-6$ ) and $0.6 \pm 1.05$ (range $0-5$ ) respectively. Overall, the breakdown of scores was: poor $(\mathbf{7 8 . 1 \%})$, moderate $(20.3 \%)$ and satisfactory $(6.2 \%)$ for signs/symptoms and poor $(\mathbf{9 3 . 2 \%})$, moderate $(\mathbf{6 . 2 \%})$ and satisfactory $(0.7 \%)$ for risk factors. Higher level of education, female gender and non-Malay race were associated with higher scores for both signs/symptoms and knowledge of screening modality; however the overall scores were low. Conclusions: Our study showed that the general knowledge of CRC in Brunei Darussalam is poor. Being female, with higher levels of education and non-Malay race were associated with higher scores, but they were still generally poor. More needs to be done to increase the public knowledge and awareness of CRC.
\end{abstract}

Keywords: Brunei - colorectal cancer - knowledge - symptoms - screening

Asian Pac J Cancer Prev, 16 (9), 3927-3930

\section{Introduction}

Colorectal cancer (CRC) is the most common gastrointestinal cancers and the incidence is increasing, particularly in the developing nations (IARC, 2012; Somi et al., 2014), including Brunei Darussalam (Chong et al., 2009; 2014). In some of the developed nations of Northern Europe and Northern America, the incidence rates are actually declining, due in part to the effect of CRC screening (IARC 2012; Austin et al., 2014). With screening, CRC are being detected early and prevented through the removal of premalignant lesions. However, uptakes of screening programs remain suboptimal. In many developing nations including Brunei Darussalam, no such formal programs are available partly because of cost and manpower issues. In places where no formal screening programs are available, opportunistic screenings are being carried out.

There are many barriers to screening and some of these include disparities in healthcare coverage, infrastructures, social divides, economic and financial factors, clinician practices, and poor awareness and knowledge of CRC (Gimeno García et al., 2011; Jerant et al., 2014; Menvielle et al., 2014; Norwati R et al., 2014;
Tripathi et al., 2014). Awareness and knowledge are important in the management of any medical disorders, and also the success of any screening programs. With the increasing and ageing population, increase in chronic noncommunicable diseases (NCDs) and escalating healthcare cost, it is becoming more important for the public to be cognizant of their health. Awareness of the signs and symptoms of common disorders should lead to earlier presentations and diagnosis; and favourable treatment outcomes. Studies have reported poor knowledge and awareness of CRC in both developed and developing nations. Such findings are not restricted to CRC but also other cancers and other medical disorders (Deepa et al., 2014; Lundelin et al., 2014; Menvielle et al., 2014; Roomizadeh et al., 2014). Therefore, understanding the background knowledge levels of the public is important and can allow formulation of measures to raise the awareness. This study assessed the level of knowledge and awareness of CRC in Brunei Darussalam.

\section{Materials and Methods}

\section{Subjects}

This study was a cross-sectional study questionnaire.

${ }^{1}$ Division of Gastroenterology, Department of Medicine, ${ }^{2}$ Department of Renal Medicine, ${ }^{3}$ Department of Surgery, RIPAS Hospital, Brunei Darussalam*For correspondence: chongvuih@yahoo.co.uk 
Subjects which consisted of patients or accompanying relatives attending the various outpatient clinics in a tertiary hospital were randomly approached and invited to participate in this interview study. Explanations were given regarding the purpose of the study and verbal consents were obtained from those who agreed to participate.

\section{Data collected}

Demographic data (age, gender, race, level of education and marital status), family history of CRC, previous CRC screening and were asked on signs/ symptoms and risk factors for CRC and also the screening modalities they were aware off. The questionnaire used was based on the questionnaire used by the Asia Pacific Colorectal Cancer Working, but modified to suit our population and aims of the study (Khoo et al., 2012). Interviews were conducted by researchers proficient in both the English or Malay languages depending on the preferences of the subjects. Subjects were asked to name as many signs/symptoms and risk factors of CRC that they were aware of. Predefined CRC symptoms and signs include; altered bowel habit, bleeding per rectum, abdominal pain, passage of mucus, incomplete defeacation or feeling of incomplete evacuation (tenesmus), anemia, weight loss, lethargy, diarrhea, constipation and vomiting. Predefined risk factors include; increasing or old age, male gender, family history or CRC or any cancer, diets low in fiber, high in meat, fatty food or fried food, being overweight, low exercise, smoking and presence of bowel disease. Any descriptions given by the subjects that fit or described the predefined answers were accepted as correct answers with further clarifications. Subjects were given the opportunities to revisit individual sections so that they could volunteer further answers. Subjected were also asked periodically and at the end of the interview if there were further answers they wish to volunteer. They were later asked if they have heard of screening modalities that are available in our setting (endoscopy; colonoscopy or sigmoidoscopy, radiological imaging; CT colography, and fecal occult blood test). The types of tests were briefly explained to subjects so that they understood what each tests were.

The number of correct answers for signs/symptoms (range 0-10) and risk factors (0-10) were added up and the mean calculated. The total scores were categorised into poor (score of between 0-2), moderate (3-4) and satisfactory (5-10).

Statistical analyses: Analyses were carried using the SPSS version 16.0. Comparisons were made for the scores between genders, race (Malay and non-Malay), levels of education and marital status. A p value of less than 0.05 was taken as significant.

\section{Results}

In total, 423 subjects participated in this study. The demography of the subjects is shown in Table 1 . There were more male and most were in the 40-49 age group. The racial breakdown is consistent with the national breakdown with predominantly Malays. The majority had formal education and a third had tertiary education. All the subjects were in employment and most were married. $8.3 \%$ had family history of CRC and $8.5 \%$ has had screening

Table 1. Sociodemographic Characteristics of Subjects

\begin{tabular}{llr}
\hline Variable & & $\mathrm{N}(\%)$ \\
\hline Gender & Male & $262(61.9)$ \\
& Female & $161(38.1)$ \\
Ethnicity & Malay & $328(77.5)$ \\
& Chinese & $72(17.0)$ \\
Age group & Others & $23(5.5)$ \\
& $30-39$ & $122(29.9)$ \\
& $40-49$ & $166(39.2)$ \\
& $50-59$ & $114(26.9)$ \\
Education level & $>60$ & $21(5.0)$ \\
& No formal education & $11(2.6)$ \\
& Primary education & $36(11.1)$ \\
& Secondary education & $237(56.0)$ \\
Marital status & Tertiary education & $139(32.9)$ \\
& Married & $372(87.9)$ \\
Employment status & Single & $39(9.2)$ \\
& Others (widowed/divorced) & $12(2.8)$ \\
& Employed & $423(100)$ \\
Previous screening & Full time & $374(88.4)$ \\
Family history of CRC & & $49(11.6)$ \\
Smoke & & $36(8.5)$ \\
\hline
\end{tabular}

Table 2. Knowledge of Signs/Symptoms and Risk Factors of CRC

\begin{tabular}{lrr}
\hline Symptoms & $\mathrm{n}(\%)$ \\
\hline Altered bowel habit (change in bowel habit) & $35(8.3)$ \\
Passage of blood with stool & $96(22.7)$ \\
Passage of mucus & $2(0.5)$ \\
Diarrhoea and constipation & $82(19.4)$ \\
Abdominal pain & $138(32.6)$ \\
Anemia (low blood count) & $7(1.7)$ \\
Weight loss & $22(5.2)$ \\
Malaise (feeling tired most of the time) & $27(6.4)$ \\
Vomiting & $33(7.8)$ \\
Others not listed & $81(19.1)$ \\
Risk factors & \\
Old age & $9(2.1)$ \\
Male gender & $2(0.5)$ \\
Family history of CRC/or any cancer & $35(8.3)$ \\
Low fibre diet & $42(9.9)$ \\
High meat intake & $20(4.7)$ \\
High intake of fatty food & $59(13.9)$ \\
High intake of fried food & $21(5.0)$ \\
Being overweight & $11(2.6)$ \\
Low exercise & $10(2.4)$ \\
Smoking & $50(11.8)$ \\
Any bowel disease & $11(2.6)$ \\
\hline
\end{tabular}

Table 3.Awareness of the Available Screening Modality

\begin{tabular}{lr}
\hline Screening modality & $\mathrm{n}(\%)$ \\
\hline Fecal occult blood & $8(1.9)$ \\
Endoscopy & \\
$\quad$ Colonoscopy & $25(5.9)$ \\
$\quad$ Sigmoidoscopy & $0(0.0)$ \\
Imaging & $1(0.2)$ \\
\hline
\end{tabular}


Table 4. Comparisons of Knowledge of between the Different Variables

\begin{tabular}{|c|c|c|c|c|c|}
\hline & & $\begin{array}{l}\text { Mean score } \\
\text { symptoms }\end{array}$ & $\mathrm{p}$ value & $\begin{array}{l}\text { Mean score } \\
\text { risk factors }\end{array}$ & $\mathrm{p}$ value \\
\hline \multirow[t]{2}{*}{ Gender } & Male & $1.2(1.3)$ & 0.088 & $0.6(1.0)$ & 0.243 \\
\hline & Female & $1.4(1.5)$ & & $0.7(1.1)$ & \\
\hline \multirow[t]{2}{*}{ Ethnicity } & Malay & $1.2(1.3)$ & 0.088 & $0.5(0.9)$ & $<0.001$ \\
\hline & Others & $1.5(1.5)$ & & $1.1(1.4)$ & \\
\hline \multirow[t]{4}{*}{ Age group } & $30-39$ & $1.3(1.3)$ & 0.635 & $0.6(0.9)$ & 0.852 \\
\hline & $40-49$ & $1.3(1.4)$ & ANOVA & $0.6(1.0)$ & ANOVA \\
\hline & $50-59$ & $1.3(1.5)$ & & $0.6(1.2)$ & \\
\hline & $>60$ & $1.2(1.5)$ & & $1.0(1.4)$ & \\
\hline \multirow[t]{4}{*}{ Education level } & No formal education & $0.9(1.5)$ & $<0.001$ & $0.4(0.9)$ & $<0.001$ \\
\hline & Primary education & $0.7(1.1)$ & ANOVA & $0.5(1.0)$ & ANOVA \\
\hline & Secondary education & $1.1(1.4)$ & & $0.4(0.8)$ & \\
\hline & Tertiary education & $1.8(1.4)$ & & $1.1(1.3)$ & \\
\hline \multirow[t]{3}{*}{ Marital status } & Married & $1.3(1.4)$ & 0.538 & $0.63(1.0)$ & 0.701 \\
\hline & Single & $1.2(1.2)$ & ANOVA & $0.7(1.1)$ & ANOVA \\
\hline & Others (widowed/divorced) & $1.7(1.8)$ & & $0.8(1.4)$ & \\
\hline \multirow[t]{3}{*}{ Employment status } & Employed & & & & \\
\hline & Full time & $1.3(1.4)$ & 0.265 & $0.6(1.0)$ & 0.089 \\
\hline & Part time & $1.5(1.4)$ & & $0.9(1.2)$ & \\
\hline \multirow{2}{*}{ Family history of CRC } & Yes & $1.7(1.5)$ & $<0.001$ & $1.0(1.3)$ & $<0.001$ \\
\hline & No & $1.0(1.3)$ & & $0.5(0.8)$ & \\
\hline
\end{tabular}

procedures done for CRC.

Overall, $54.1 \%$ could not name any signs/symptoms or risk factors for CRC. $43.3 \%$ could name any signs/ symptoms and $63.1 \%$ could name any risk factors of CRC. However, the overall knowledge of the individual symptoms and risk factors were very poor (Table 2). The overall score for CRC symptoms and risk factors were $1.3 \pm 1.39$ (range $0-6$ ) and $0.6 \pm 1.05$ (range 0-5). Overall, the breakdowns of score were poor $(78.1 \%)$, moderate (20.3\%) and satisfactory $(6.2 \%)$ for signs/symptoms and poor $(93.2 \%)$, moderate $(6.2 \%)$ and satisfactory $(0.7 \%)$ for risk factors.

With regards to screening modalities for CRC, the overall awareness was poor (Table 4). Comparing the different variables, there were significant differences in the mean scores in ethnicities, education levels and family history of CRC (Table 4).

\section{Discussion}

In this study, we showed that the level of knowledge of CRC in our population was generally poor. Overall, half of our subjects were not able to name any symptoms or risk factors for CRC. Only $26.5 \%$ had scores of moderate to satisfactory for signs/symptoms and 6.9\% achieved this score for risk factors of CRC. Despite the overall poor knowledge and awareness, there were some differences (Table 4). Non-Malay ethnicities had higher mean scores for both signs/symptoms and risk factors, significant for the latter $(\mathrm{p}<0.001)$. As expected, the mean scores improved with increasing levels of education, significantly higher among those with tertiary education compared to the other groups. However, the overall mean scores were low. There were no differences between the genders, different age groups, marital status and working status. Even in subjects with family history of CRC, the mean scores were low, but were significantly better than the group without family history of CRC.
Low level of knowledge and awareness of CRC is common and is not restricted to the developing or underdeveloped nations. Studies from developed nations have all shown suboptimal knowledge and awareness for CRC, although better than developing nations (Khoo et al., 2012). A recent multi-centre Asia Pacific study reported low knowledge in countries with low participation in CRC screening. The more developed nations like Australia, Singapore and Hong Kong reported higher knowledge. Similarly, poor knowledge and awareness of CRC were also common among those at risk for CRC (family history of CRC including those who are first degree relatives) (Chen et al., 2013; Andosoy et al., 2014). Studies from China (Chen et al., 2013) and Turkey (Andosoy et al., 2014) on healthcare professionals have shown that knowledge and awareness for CRC (also including breast and cervical cancer in the Turkish study) were varied from poor to good. A study from Malaysia, with a population demographic similar to ours, reported poor level of knowledge and awareness of CRC (Su et al., 2013). The authors reported that $38 \%$ and $32 \%$ respondents could not name any warning symptoms or risk factors for CRC respectively. In our study, $43.3 \%$ could name any signs/ symptoms and $63.1 \%$ could name any risk factors of CRC.

Why were the overall mean scores low, even for those who had tertiary educations and those with family history? Well known reasons for poor knowledge and awareness include lower or no education, lower incomes, ethnicities especially those from disadvantaged and certain issues related to societies (Gimeno García et al., 2011; Jerant et al., 2014; Menvielle et al., 2014; Norwati et al., 2014; Tripathi et al., 2014). Many developed nations have good public awareness campaigns that are specifically designed to improve education on CRC. This type of initiative is lacking in many developing countries due to poor support from funding bodies or participations from disease survivors. Mass media influences may not be as advanced and pervasive as developed nations. Whether 
the types of healthcare system and coverage have any impact in the level of knowledge is uncertain. However, is it possible that in a healthcare system where patients pay for treatment, either through insurance or other arrangements, the level of involvement or awareness of own health maybe higher than in system where healthcare is provided free. Brunei Darussalam has a healthcare system that provides free medical coverage to all her citizens and permanent residents. It can be deduced that patients are less inclined to be responsible for their health due to the absence of financial accountability.

Poor knowledge has many major deleterious implications. It may lead to delayed presentations, more advanced disease at diagnosis and patients declining or delay appropriate treatment (surgery, chemotherapy and radiotherapy). Lack of confidence on the available standard treatment is not uncommon and many still seek alternative 'Kampong or village' remedies. In our experience, it is not infrequent to encounter patients requesting delay in any planned interventions in order to try out alternative unproven remedies.

Apart from impacting on the course of presentations and outcomes, poor knowledge and awareness of the available screening modalities will also affect any attempts to address the common disorders. Higher knowledge has been shown to be predictor of participations in CRC screening (Khoo et al., 2012; Christou \& Thompson, 2012; Khayyat \& Ibrahim, 2014). We previously showed that screening for CRC is feasible in our setting and can be effective (Chong VH et al., 2013). This study reported CRC screening of government servants in a one-time health screening program through fecal occult blood testing, family history of CRC or personal history of CRC or colonic polyp (Chong et al., 2013). Overall, positivity for FOB was $1.5 \%$. However, like any other CRC screening program, the uptake was suboptimal with only $17.7 \%$ of those eligible attended for screening. Of those who attended, only half agreed to proceed with screening colonoscopy and $12.9 \%$ were found to have advanced lesions, including a case of carcinoma in-situ.

In order to tackle the overall poor knowledge and awareness, more public awareness programs through road shows, public lectures or forums, educational articles and debates through the various medias such newspapers, social websites, radio and television programs are needed. Inclusion of the colorectal cancer awareness event to the health promotion schedule will be important. However, it is important that such programs are sustained and carried out periodically.

In conclusion, our study showed that the overall knowledge and awareness of CRC is poor among our population, even in those whom we expected to do better, such as those with higher education and family history. Given the increasing incidence of CRC and the lack of formal screening program in our setting, more efforts are needed to improve public awareness.

\section{References}

Austin H, Jane Henley S, King J, Richardson LC, Eheman C (2014). Changes in colorectal cancer incidence rates in young and older adults in the United States, what does it tell us about screening. Cancer Causes Control, 25, 191-201.

Andsoy II, Gul A (2014). Breast, cervix and colorectal cancer knowledge among nurses in Turkey. Asian Pac J Cancer Prev, 15, 2267-72.

Chen YS, Xu SX, Ding YB, et al (2013). Colorectal cancer screening high-risk populations, a survey of cognition among medical professionals in Jiangsu, China. Asian Pac J Cancer Prev, 14, 6487-91.

Chong VH, Abdullah MS, Telisinghe PU, Jalihal A (2009). Colorectal cancer, incidence and trend in Brunei Darussalam. Singapore Med J, 50, 1085-9.

Chong VH, Bakar S, Sia R, et al (2013). Colorectal cancer screening among government servants in Brunei Darussalam. Asian Pac J Cancer Prev, 14, 7657-61.

Christou A, Thompson SC (2012). Colorectal cancer screening knowledge, attitudes and behavioural intention among Indigenous Western Australians. BMC Public Health, 12, 528.

Deepa M, Bhansali A, Anjana RM, et al (2014). Knowledge and awareness of diabetes in urban and rural India, The Indian Council of Medical Research India Diabetes Study (Phase I), Indian Council of Medical Research India Diabetes, 4, Indian J Endocrinol Metab, 18, 379-85.

Gimeno García AZ, Quintero E, Nicolás Pérez D, Hernández M, JiménezSosa A (2011). Colorectal cancer screening in first-degree relatives of colorectal cancer, participation, knowledge, and barriers against screening. Eur $J$ Gastroenterol Hepatol, 23, 1165-71.

Hilmi I, Hartono JL, Goh K (2010). Negative perception in those at highest risk--potential challenges in colorectal cancer screening in an urban asian population. Asian Pac J Cancer Prev, 11, 815-22.

Jerant A, Kravitz RL, Sohler N, et al (2014). Sociopsychological tailoring to address colorectal cancer screening disparities, a randomized controlled trial. Ann Fam Med, 12, 204-14.

Khayyat YM, Ibrahim EM (2014). Public awareness of colon cancer screening among the general population, A study from the Western Region of Saudi Arabia. Qatar Med J, 16, 17-24.

Lundelin K, Graciani A, García-Puig J, et al (2012). Knowledge of stroke warning symptoms and intended action in response to stroke in Spain, a nationwide population-based study. Cerebrovasc Dis, 34, 161-8.

Menvielle G, Richard JB, Ringa V, Dray-Spira R, Beck F (2014). To what extent is women's economic situation associated with cancer screening uptake when nationwide screening exists? A study of breast and cervical cancer screening in France in 2010. Cancer Causes Control, 25, 977-83.

Norwati D, Harmy MY, Norhayati MN, Amry AR (2014). Colorectal cancer screening practices of primary care providers, results of a national survey in Malaysia. Asian Pac J Cancer Prev, 15, 2901-4.

Roomizadeh P, Taheri D, Abedini A, et al (2014). Limited knowledge of chronic kidney disease and its main risk factors among Iranian community, an appeal for promoting national public health education programs. Int J Health Policy Manag, 2, 161-6.

Somi MH, Golzari M, Farhang S, Naghashi S, Abdollahi L (2014). Gastrointestinal cancer incidence in East Azerbaijan, Iran, update on 5 year incidence and trends. Asian Pac J Cancer Prev, 15, 3945-9.

Su TT, Goh JY, Tan J, et al (2013). Level of colorectal cancer awareness, a cross sectional exploratory study among multiethnic rural population in Malaysia. BMC Cancer. 13, 376.

Tripathi N, Kadam YR, Dhobale RV, Gore AD (2014). Barriers for early detection of cancer amongst Indian rural women. South Asian J Cancer, 3, 122-7. 